\title{
Dynamics of Commensalism Interaction with Linear and Holling Type II Functional Responses by the Host to the Commensal Species
}

\author{
Geremew K ${ }^{1}$, Prof. N. Phani Kumar ${ }^{2}$, K. V. L. N. Acharyulu ${ }^{3}$ and Boka Kumsa ${ }^{4}$ \\ ${ }^{1,2,4}$ Faculty, Department of Mathematics, \\ College of Natural and Computational Sciences, Nekemte, Ethiopia \\ ${ }^{3}$ Associate Professor, Department of Mathematics, Bapatla Engineering College, \\ Andhra Pradesh, India \\ kvlna@yahoo.com
}

\begin{abstract}
Population dynamics studies the changes in size and composition of populations through time as well as the biotic and abiotic factor influencing those changes. In this study, we provide a mathematical framework for Ordinary differential equation in various models with analytical and numerical study. Furthermore, in this paper a mathematical model of commensalism between two species is investigated. In this investigation we considered both linear or Holling type I and Holling type II functional responses by the host to the commenasal species. The commensal species draw strength from the host species. This model is characterized by a pair of first order non-linear coupled autonomous systems of differential equation. Three main assumptions of mathematical models are considered. All the equilibrium points of the model are identified and criteria for the stability are discussed.
\end{abstract}

Keywords: commensal, host, equilibrium point, stability analysis, phase plane analysis, Holling type II

\section{Introduction}

Ecology, basically the study of the inter relationship between species and their environment, in such areas as predator-prey and competition interactions, renewable resources management, evolution of pesticide resistant strains ecological and genetically engineered control of pests, multi-species societies, plant-herbivore systems and so on is now an enormous field. It is the scientific study of the interactions between organisms and their environment. Organisms compete with other organisms for resources (food, water, space). They eat and are eaten by other organisms [6]. The study of population change has a very long history [2]; in 1202 an exercise in an arithmetic book written by Leonard Pisa involved building a mathematical model for a growing rabbit population. Mathematical modeling is so an important interdisciplinary activity which involves the study of some aspects of diverse disciplines. Mathematical modeling of ecosystem was initiated by Malthus (exponential growth) [3-4] Verhulst (Logistic growth) and Lotka-Voltera [4]. Since then several mathematicians and ecologists contributed to the growth of this area of knowledge [6]. In general, there is a whole web of interacting species, sometimes called atrophic web, which makes for structurally complex communities. We consider here systems involving two or more species, concentrating particularly on two species systems.

Now, the present investigation is a study of a continuous model of "a symbiotic interaction called Commensalism" between two species. Commensalism in biology is a relation between individuals of two species in which one species obtains food or other benefits from the other without either harming or benefiting the latter. The commensal- 
the species that benefits from the association-may obtain nutrients, shelter, support, or locomotion from the host species, which is unaffected. The commensal relation is often between a larger host and a smaller commensal. The host organism is essentially unchanged by the interaction, whereas the commensal species may show great morphological adaptation.

One of the best-known examples of a commensal is the remora (family Echineidae) that rides attached to sharks and other fishes. Remoras have evolved on the top of their heads a flat oval sucking disk structure that adheres to the bodies of their hosts. Both remoras and pilot fishes feed on the leftovers of their hosts' meals.

Other examples of Commensal include bird species, such as the great egret (Ardea $a l b a$ ), that feed on insects turned up by grazing mammals or on soil organisms stirred up by plowing. Various biting lice, fleas, and louse flies are Commensal in that they feed harmlessly on the feathers of birds and on sloughed-off flakes of skin from mammals. That is cattle egrets feast on insects that area roused in to flight by cattle grazing in the insects' habitat. The cattle do not benefit at all.

The differential equations are very much helpful in many areas of science. But most of the interesting real life problems involve more than one unknown function. Therefore, the use of system of differential equations is very useful [1]. Without loss of generality, we will concentrate on systems of two differential equations. Non linear phenomena are woven into the fabric of biological systems. Interactions between individuals, species, or populations lead to relationships that depend on the variables. That means models proportion to describe such phenomena contain nonlinear equations [2]. The model equations for two species host -Commensal are given by first order non -linear systems of differential equations; since most continuous models of population dynamics are based on differential equations which can be solved using a variety of techniques which provide information regarding equilibrium and their stability [6].

In this paper, a comparative study of three models is considered relative to the interaction of commensalism type between the two species. We consider the following three assumptions and models. (i). First assumption in Model 1(A): Involvements of Basic host commensal interaction. (ii). Second assumption in Model-2(B): Active role of Carrying capacity and (iii). Third assumption in model-3(C): Incorporation of functional response to the species. In model-1(A) the species have an exponential growth which is not realistic in nature. In model $-2(\mathrm{~B})$ and model $-3(\mathrm{C})$ have logistic growth rate. These models are more complex than model-1(A). Even model-3(C) is more realistic than the other two models in the sense that the commensalism is characterized by a function of host species which is not linear. In model-2(B) the commensal species grows up to the carrying capacity and decline in the absence of host species [6]. The survival of this species is because of the interaction of the host species. The investigation of these assumptions will be given in detail in the following section.

\section{Assumptions and Basic Models Equations}

In this paper, we describe the two species host -Commensal model. To develop this model, the assumptions have been made as mentioned.

i. $H(t)$ and $C(t)$ denote the density of Host and Commensal respectively at any instant of time $\mathrm{t}$ subject to the non-negative initial conditions $H(0)=H_{0} \geq 0$ and $C(0)=C_{0} \geq 0$.

ii. The parameters $r_{i}$ and $k_{i}, \mathrm{i}=1,2$ be the natural growth rates and the carrying capacities of commensal population and host population respectively, and their growth depends on the assumption of the models. 
iii. The parameter $a$ is the commensal coefficient of $C(t)$ due to the host $H(t)$ i.e the helping rate of host to the commensal or the rate at which the host helping to the commensal.

iv. The term $\frac{a C}{1+a C h}$ is helping rate by the host and $C$ denotes commensal (taking help from the host) density. This represents the functional response for commensal species by host species. This functional response called Holling type-II functional response represents the rate at which the commensal benefited, given that the host needs on average $\mathrm{h}$ time units to handle the species of commensal item. The type II functional response is hence based on the assumption that at very host species densities, commensal species become abundant by their handling time.

v. The parameter(h) is average time of response [handling (helping) time] of the host. Under the above-mentioned assumptions the model equations for two species host Commensal are given by the following first order non -linear systems of differential equations.

\section{A Basic Host-Commensal interaction(linear response)}

The first model considered is the interaction of the two species which can be described by exponential growth. Suppose $C=C(t)$ and $H=H(t)$ represents the size of the population of the commensal and host species respectively at any time $t$ and $r_{1}$ and $r_{2}$ is the natural growth rate of the species; $a$ is the commensal coefficient of commensal $\mathrm{C}$ due to host $\mathrm{H}$, provided that all the parameters are non-negative constants. The basic Host-commensal interaction of the two species was given by the following differential equations;

$$
\begin{aligned}
& \frac{d C}{d t}=r_{1} C+a C H \\
& \frac{d H}{d t}=r_{2} H
\end{aligned}
$$

The only equilibrium point for this basic host-commensal interaction is the trivial one which is $\left(C^{*}, H^{*}\right)=(0,0)$. Thus far we have arrived at one steady state solution that satisfies the system. In realistic situations there are always small disturbances. Thus it is of interest to determine whether such deviations from the steady state will lead to drastic changes or will be damped out.

The variation matrix at the steady state is $\left(\mathrm{C}^{*}, \mathrm{H}^{*}\right) ; \mathrm{Jl}_{\left(\mathrm{C}^{*}, \mathrm{H}^{*}\right)}=\left(\begin{array}{cc}\mathrm{r}_{1} & 0 \\ 0 & \mathrm{r}_{2}\end{array}\right)$, where $\mathrm{r}_{1}, \mathrm{r}_{2}>$ 0 . The eigen values of this variation matrix are positive; hence the species has no stability. This can be analyzed by employing linearization.

The basic model we have seen is not considered structurally, since the response is linear that a small change to its structure does not cause a significant change in its predictions. In another words the above model is not relevant from the biological point of view. That is, in nature it is impossible for population to grow exponentially because there are different factors that affect the population to grow exponentially.

\section{B. The Host-Commensal Interaction Model in which both the Species Grow Logistically}

Now, the second model is concerned with incorporating a carrying capacity to both species the host-commensal interaction which is described as;

$$
\begin{gathered}
\frac{d C}{d t}=r_{1} C\left(1-\frac{C}{k_{1}}\right)+a C H \\
\frac{d H}{d t}=r_{2} H\left(1-\frac{H}{k_{2}}\right)
\end{gathered}
$$


2.B.1. Existence of Equilibrium points: To study the stability of the assumed model, the equilibrium points or steady states in the system (1.2) are mandatory to calculate. Hence, the system under investigation has the following steady-state given by;

(i) Trivial equilibrium point in which both interactions are not yet exist. $E_{0}(0,0)$ is one equilibrium point

(ii) The case when the host survives and the Commensal is not, that is $E_{1}\left(0, k_{2}\right)$ is another equilibrium point

(iii) The case when the Commensal survive and the host does not exist; $E_{2}\left(k_{1}, 0\right)$ is another equilibrium point

(iv) The situation in which the interaction of the two species exists; i.e., co-existence happens. That is $E_{3}\left(\frac{k_{1}}{r_{1}}\left(r_{1}+a k_{2}\right), k_{2}\right)$ is another equilibrium point in which the Commensal flourishes.

\section{C. Host-Commensal Interaction (Assumption with Functional Responses)}

The third assumption is more generalized than the above assumption in the sense that the commensal coefficient is not linear function rather Monod function $G(C)=\frac{a C}{1+a C h}$. The Mathematical model of this interaction is given by;

$$
\begin{gathered}
\frac{d C}{d t}=r_{1} C\left(1-\frac{C}{k_{1}}\right)+\frac{a C}{1+a C h} H \\
\frac{d H}{d t}=r_{2} H\left(1-\frac{H}{k_{2}}\right)
\end{gathered}
$$

Where; $\mathrm{h}$ - helping time and a-helping rate

Now let's incorporate this idea with host- commensal interaction.

The equation $G(C)=\frac{a C}{1+a C h}$ is called host functional response. Where $G$ denotes helping rate by the host and $C$ denotes commensal (taking help from the host) density. The rate at which the host helping to the commensal is called the helping rate, $a$. The average time of help for the host is called the handling (helping) time; $h$.

To analyze this model, we apply non- dimensionalization

The system becomes;

$$
\begin{gathered}
\frac{d C}{d t}=C-C^{2}+\frac{\beta C}{1+\alpha C} H \\
\frac{d H}{d t}=r H-H^{2} \quad, \beta, \alpha \text { and } r
\end{gathered}
$$

are parameters after non-dimensionalization.

2.C.1. Existence of Equilibrium Points: Now the steady states of the system are given by; $\frac{d H}{d t}=0$ and $\frac{d C}{d t}=0$. Hence the system (1.4) possesses the following steady states. $\boldsymbol{S}_{\mathbf{0}}(\mathbf{0}$, $0), S_{1}(1,0), S_{2}(0,1)$ and $S_{3}\left(\frac{(\alpha-1)+\sqrt{\delta}}{2 \alpha}, 1\right)$ where $\delta=(1-\alpha)^{2}+4(\alpha)(1+\beta)>0$

\section{Stability Analysis}

Let us study the stability criteria of deterministic differential equation $(1.2,1.3 / 1.4)$ around the equilibrium point $E\left(C^{*}, H^{*}\right)$ assumption of the model with equation (1.2) and $S\left(C^{*}, H^{*}\right)$ with equation (1.3/1.4). This stability is obtained by the nature of the eigenvalues of the Jacobian matrix (community matrix) at that equilibrium point; the community matrix of the differential equation (1.2) and (1.3/1.4) is given by;

Then Jacobian matrix (community matrix) 


$$
\mathrm{J}:=\left(\begin{array}{ll}
\frac{\partial f}{\partial C} & \frac{\partial f}{\partial H} \\
\frac{\partial g}{\partial C} & \frac{\partial g}{\partial H}
\end{array}\right)=\left(\begin{array}{cc}
r_{1}-\frac{2 r_{1} C}{k_{1}}+a H & a C \\
0 & r_{2}-\frac{2 r_{2 H}}{k_{2}}
\end{array}\right)
$$

Jacobian matrix of the system $\mathrm{J}=$

$$
:=\left(\begin{array}{cc}
\frac{\partial f}{\partial C} & \frac{\partial f}{\partial H} \\
\frac{\partial g}{\partial C} & \frac{\partial g}{\partial H}
\end{array}\right)=\left(\begin{array}{cc}
1-2 C+\frac{(\beta H)(1+\alpha C)-(\beta C H)(\alpha)}{(1+\alpha C)^{2}} & \frac{\beta C}{1+\alpha C} \\
0 & r-2 r H
\end{array}\right)
$$

Next, summarizing the stability of the equilibrium state using the nature of eigenvalues with the corresponding eigenvectors of the variational matrix (Jacobian matrix) around the steady states are given in the following Table (1).

Table 1. Summary of Steady States where $M=-\frac{\left(\mathbf{a k}_{1}+\frac{\mathbf{a}^{2} \mathbf{k}_{1} \mathbf{k}_{2}}{\mathbf{r}_{1}}\right)}{\mathbf{r}_{2}-\left(\mathbf{r}_{1}+\mathbf{k}_{2}\right)}<0$

\begin{tabular}{|l|l|c|c|c|}
\hline Critical point & $E_{0}(0,0)$ & $E_{1}\left(k_{1}, 0\right)$ & $E_{2}\left(0, k_{2}\right)$ & $E_{3}\left(\left(\frac{k_{1}}{r_{1}}\left(r_{1}+a k_{2}\right), k_{2}\right)\right.$ \\
\hline Jacobian & $\left(\begin{array}{cc}r_{1} & 0 \\
0 & r_{2}\end{array}\right)$ & $\left(\begin{array}{cc}-r_{1} & 0 \\
0 & r_{2}\end{array}\right)$ & $\left(\begin{array}{cc}r_{1}+a k_{2} & 0 \\
0 & -r_{2}\end{array}\right)$ & $\left(\begin{array}{cc}-r_{1}-a k_{2} & a k_{1}+\frac{a^{2} k_{1} k_{2}}{r_{1}} \\
0 & -r_{2}\end{array}\right)$ \\
\hline Eigen values & $\lambda_{1,2}=r_{1}, r_{2}$ & $\lambda_{1,2}=-r_{1}, r_{2}$ & $\lambda_{1,2}=-r_{2},\left(r_{1}+a k_{2}\right)$ & $\lambda_{1,2}=-r_{2},-\left(r_{1}+a k_{2}\right)$ \\
\hline Eigenvector & $v_{1}=\left(\begin{array}{l}1 \\
0\end{array}\right)$ & $v_{1}=\left(\begin{array}{l}1 \\
0\end{array}\right)$ & $v_{1}=\left(\begin{array}{l}1 \\
0\end{array}\right)$ & $v_{1}=\left(\begin{array}{c}M \\
1\end{array}\right)$ \\
$v_{2}=\left(\begin{array}{l}0 \\
1\end{array}\right)$ & $v_{2}=\left(\begin{array}{l}0 \\
1\end{array}\right)$ & $v_{2}=\left(\begin{array}{l}0 \\
1\end{array}\right)$ & Stable (nodal sink);coexistence \\
\hline $\begin{array}{l}\text { Classification } \\
\text { of fixed points }\end{array}$ & $\begin{array}{l}\text { Unstable } \\
\text { (nodal source) }\end{array}$ & saddle & saddle & \\
\hline
\end{tabular}

Similarly, for system (1.3/1.4) we have the following summary to analyze the stability of the steady states.

Table 2. Summary of Steady States of the System (1.3)

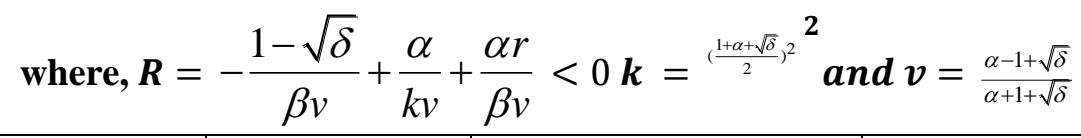

\begin{tabular}{|l|c|c|c|c|}
\hline Critical point & $(0,0)$ & $(1,0)$ & $(0,1)$ & $\left(\frac{\alpha-1+\sqrt{\delta}}{2 \alpha}, 1\right)$ \\
\hline Jacobian & $\left(\begin{array}{ll}1 & 0 \\
0 & r\end{array}\right)$ & $\left(\begin{array}{cc}-1 & \frac{\beta}{1+\alpha} \\
0 & r\end{array}\right)$ & $\left(\begin{array}{cc}1+\beta & 0 \\
0 & -r\end{array}\right)$ & $\left(\begin{array}{c}\frac{1-\sqrt{\delta}}{\alpha}+\frac{\beta}{k} \\
0\end{array}\right)$ \\
\hline Eigenvalues & $\lambda_{1,2}=1, r$ & $\lambda_{1,2}=-1, r$ & $\lambda_{1,2}=1+\beta,-r$ & $\lambda_{1,2}=\frac{1-\sqrt{\delta}}{\alpha}+\frac{\beta}{k},-r$ \\
\hline Eigenvector & $v 1=\left(\begin{array}{l}1 \\
0\end{array}\right)$ & $v 1=\left(\begin{array}{l}1 \\
0\end{array}\right)$ & $v 1=\left(\begin{array}{l}1 \\
0\end{array}\right)$ & $v 1=\left(\begin{array}{l}1 \\
0\end{array}\right)$ \\
$v 2=\left(\begin{array}{l}0 \\
1\end{array}\right)$ & $v 2=\left(\begin{array}{l}0 \\
1\end{array}\right)$ & $v 2=\left(\begin{array}{l}0 \\
1\end{array}\right)$ & $v 2=\left(\begin{array}{l}1 \\
R\end{array}\right)$ \\
\hline $\begin{array}{c}\text { Classification } \\
\text { of fixed points }\end{array}$ & $\begin{array}{c}\text { Unstable } \\
\text { (nodal source) }\end{array}$ & saddle & saddle & $\begin{array}{c}\text { Stable (nodal sink) } \\
\text { coexistence }\end{array}$ \\
\hline
\end{tabular}




\section{Phase Plane Analysis}

Note that, a phase portrait is a geometric representation of the trajectories of a dynamical system in the phase plane. Each set of initial conditions is represented by a different curve, or point. Phase portraits are helpful tools in studying dynamical systems. A phase portrait graph of a dynamical system depicts the system's trajectories with arrows.

For assumption of the model (1.1 and 1.2) the phase plane analyze is given in Figure 1 $\&$ Figure (2) and for (1.3/1.4) described in Figure (6).

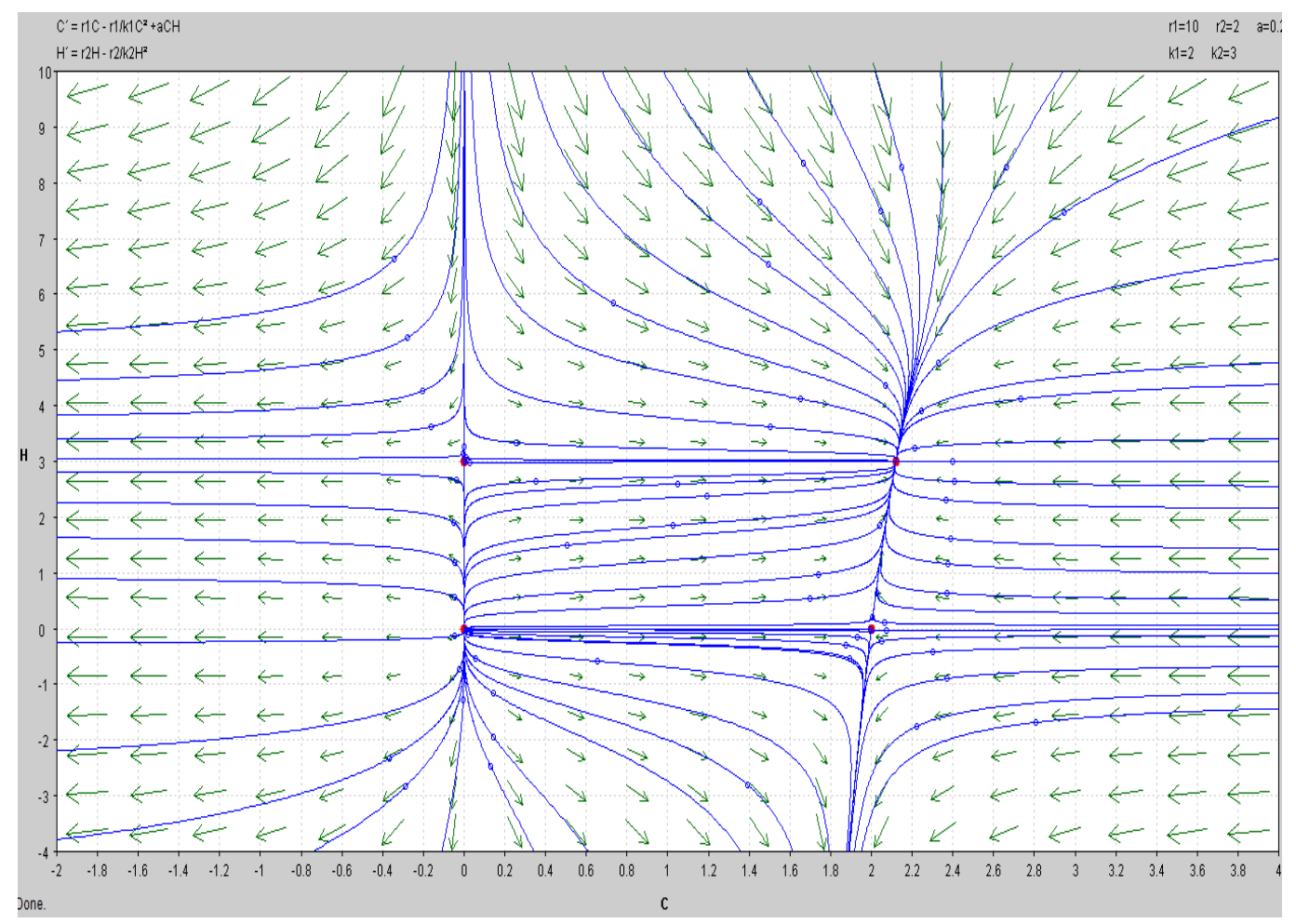

Figure 1. The Trajectories of the System with Parameters $r 1=10, r 2=2, k 1=2$, $\mathrm{k} 2=3, \mathrm{a}=0.2$

\section{Numerical Results}

In this section, we have to analyze by taking some numerical values for the set of parameters and visualizing in diagram (phase portrait).To illustrate these we use the PPlane packages. Based on the fig. 1 which is the C-H state variables, next we analyze the trajectories of the system by taking $\mathrm{C}$ versus $\mathrm{t}$ and $\mathrm{H}$-versus $\mathrm{t}$ in the next separately around the steady states by assuming a sample of trajectories. 


\section{A). For basic assumption of models 1.1and 1.2}

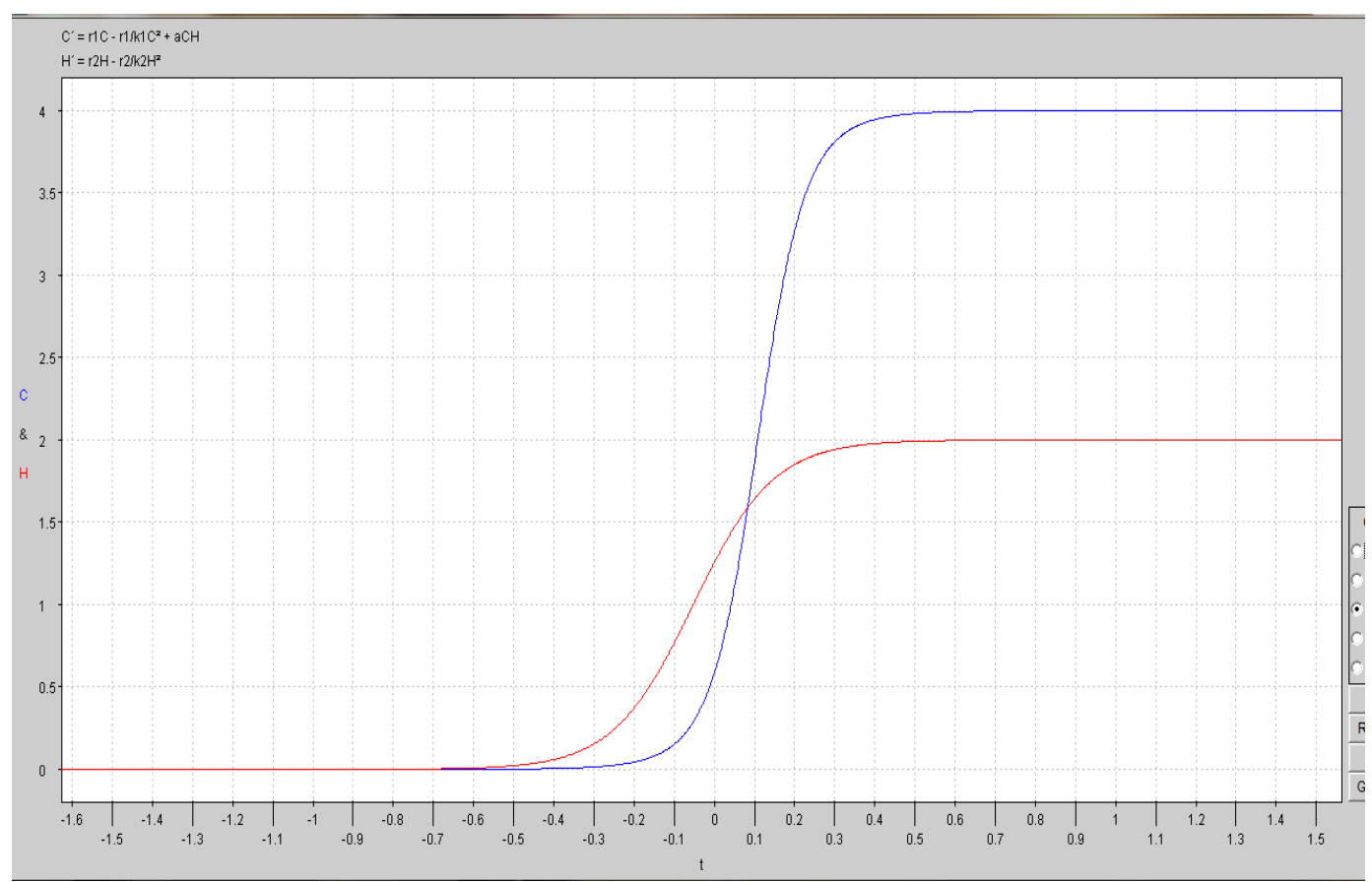

Figure 2. The Graph of the $\mathrm{C}-\mathrm{H}$ versus Time $\mathrm{t}$ around the Equilibrium Point $\mathrm{E}_{0}$

The set of parameters are $r 1=10, r 2=2, k 1=2, k 2=3, a=5$

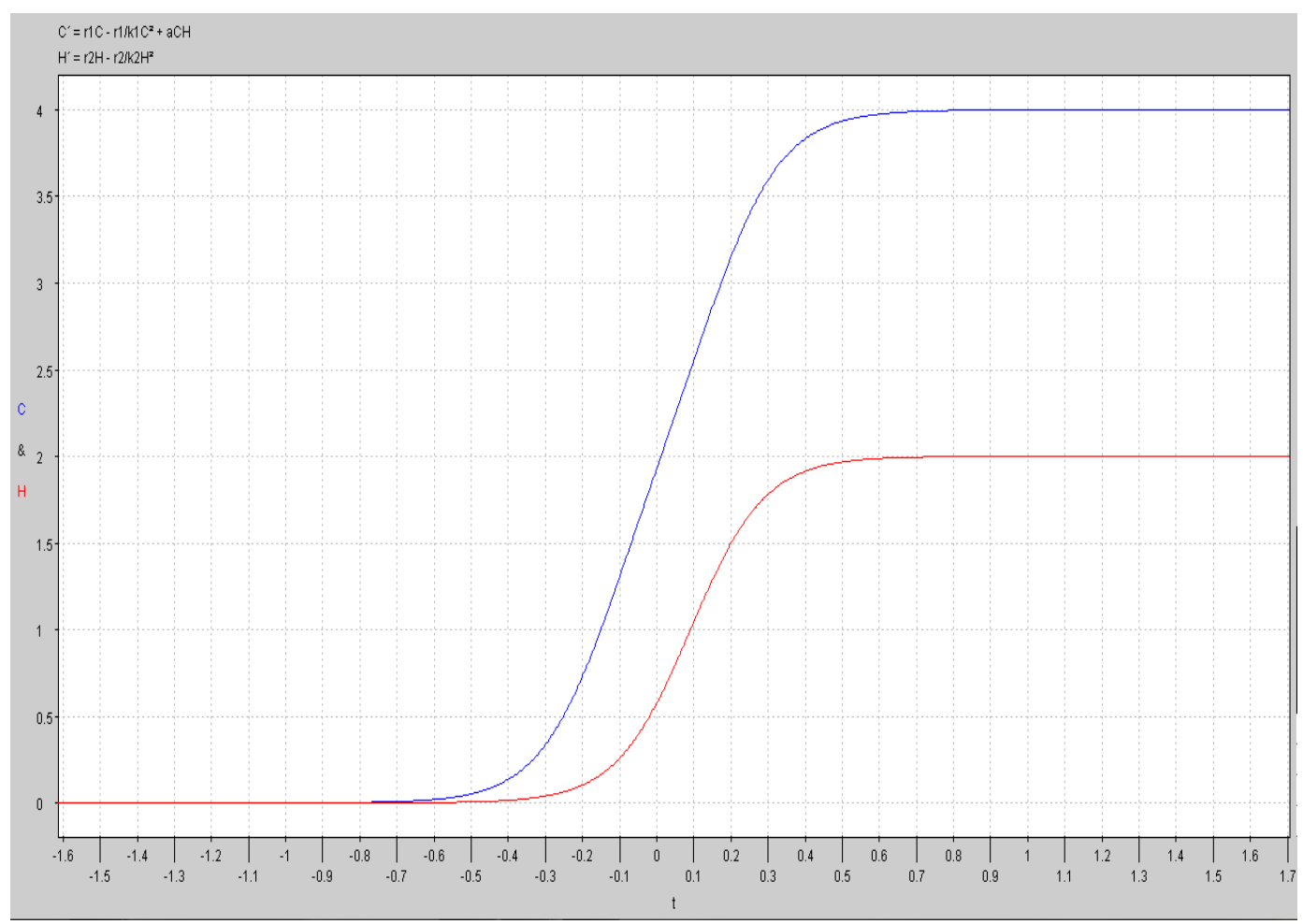

Figure 3. The Graph of the C-H with Time $t$ at the Equilibrium Point E1

The set of parameters are $r 1=10, r 2=2, k 1=2, k 2=3, a=5$ 


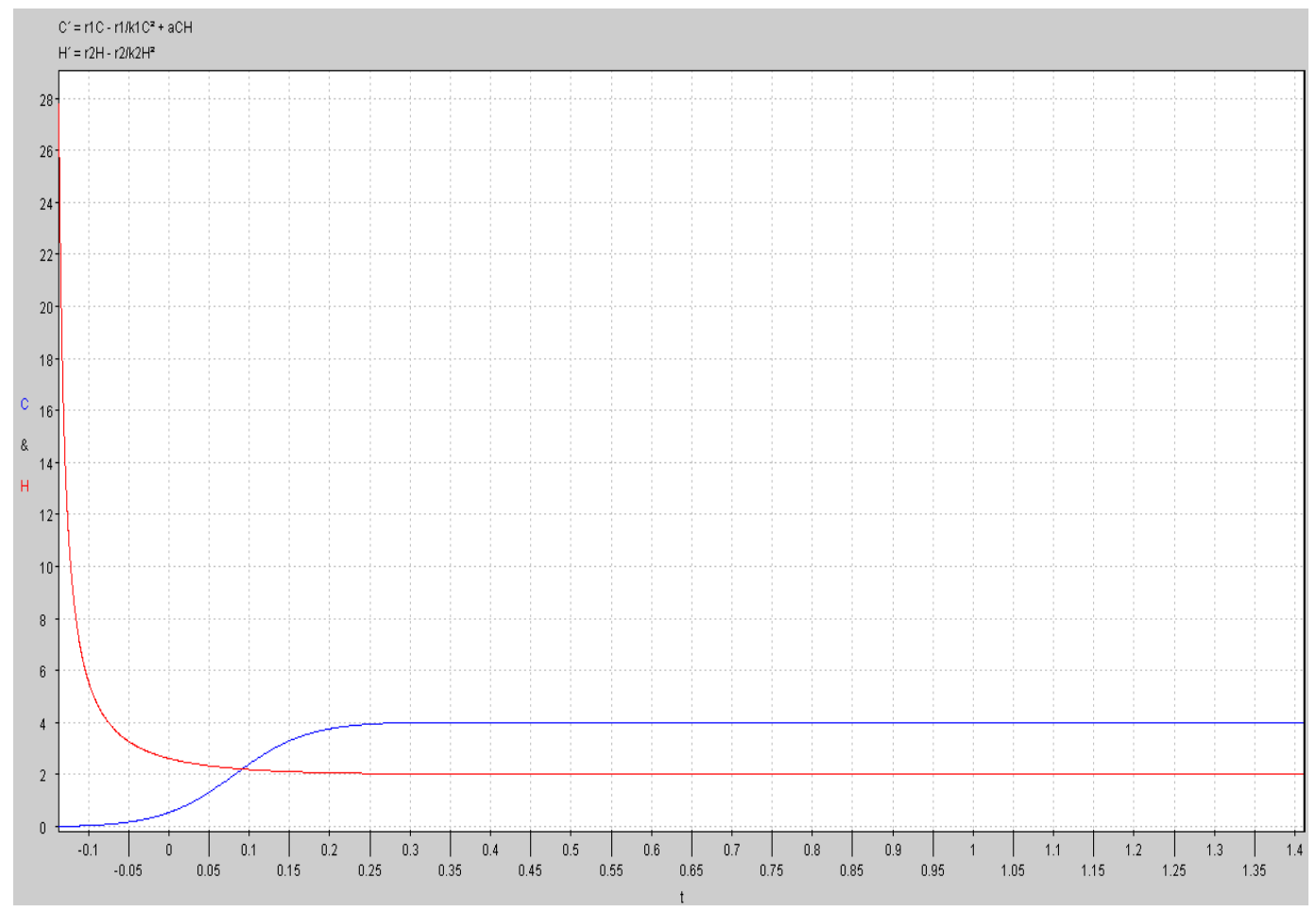

Figure 4. The Graph of the C-H with Time $t$ at the Equilibrium Point E2

The set of parameters are $r 1=10, r 2=2, k 1=2, k 2=3, a=5$

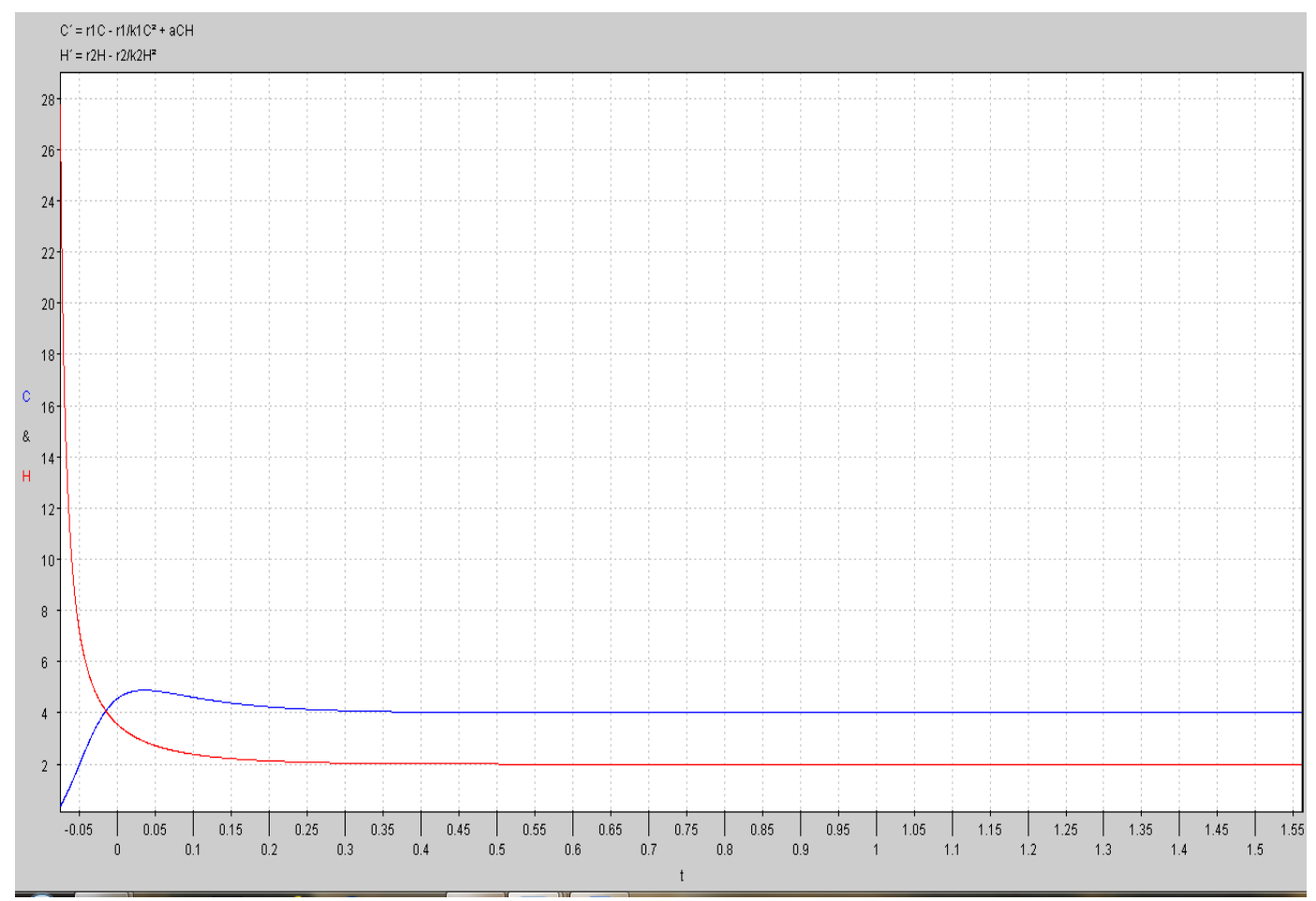

Figure 5. The Graph of $\mathrm{C}-\mathrm{H}$ versus Time $\mathrm{t}$ around the Equilibrium Point E3

The set of parameters are $r 1=10, r 2=2, k 1=2, k 2=3, a=5$ 


\section{B). For the Assumption of the System (1.3/1.4)}

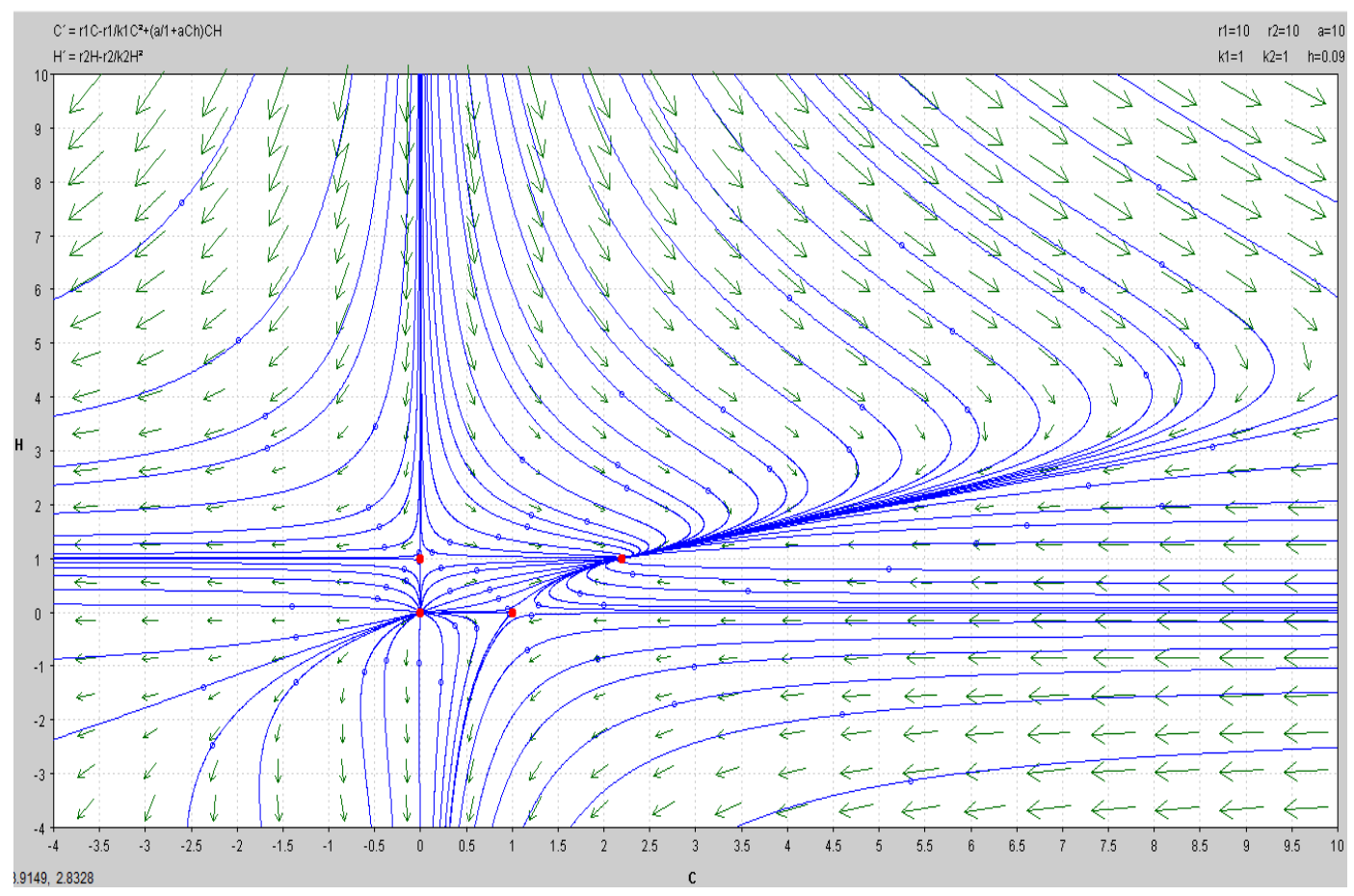

Figure 6. The Trajectories of the System with Parameters $r 1=10, r 2=10$, $k 1=1, k 2=1, a=5, h=0.09$

The phase portrait diagrams of $\mathrm{C}-\mathrm{H}$ versus time $\mathrm{t}$ around the steady states with some set of parameters are illustrated as follows.

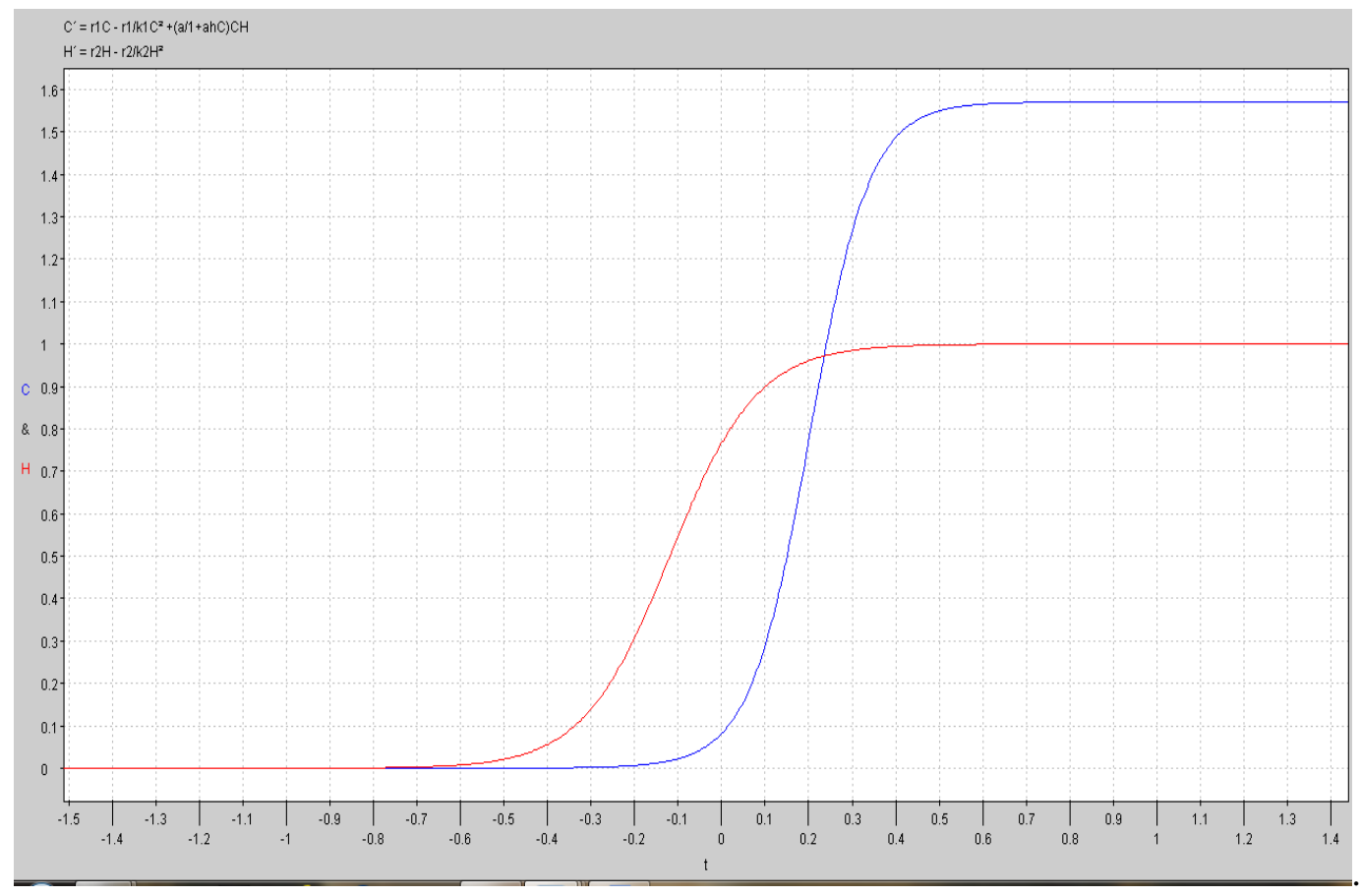

Figure 7. The Graph of the $\mathrm{C}$ - $\mathrm{H}$ with Time $\mathrm{t}$ at the Equilibrium Point S0

The set of parameters are $\mathrm{r} 1=\mathbf{1 0}, \mathbf{r} 2=\mathbf{1 0}, \mathrm{k} 1=\mathbf{1}, \mathrm{k} 2=\mathbf{1}, \mathbf{a}=\mathbf{5}, \mathrm{h}=\mathbf{0 . 0 9}$ 


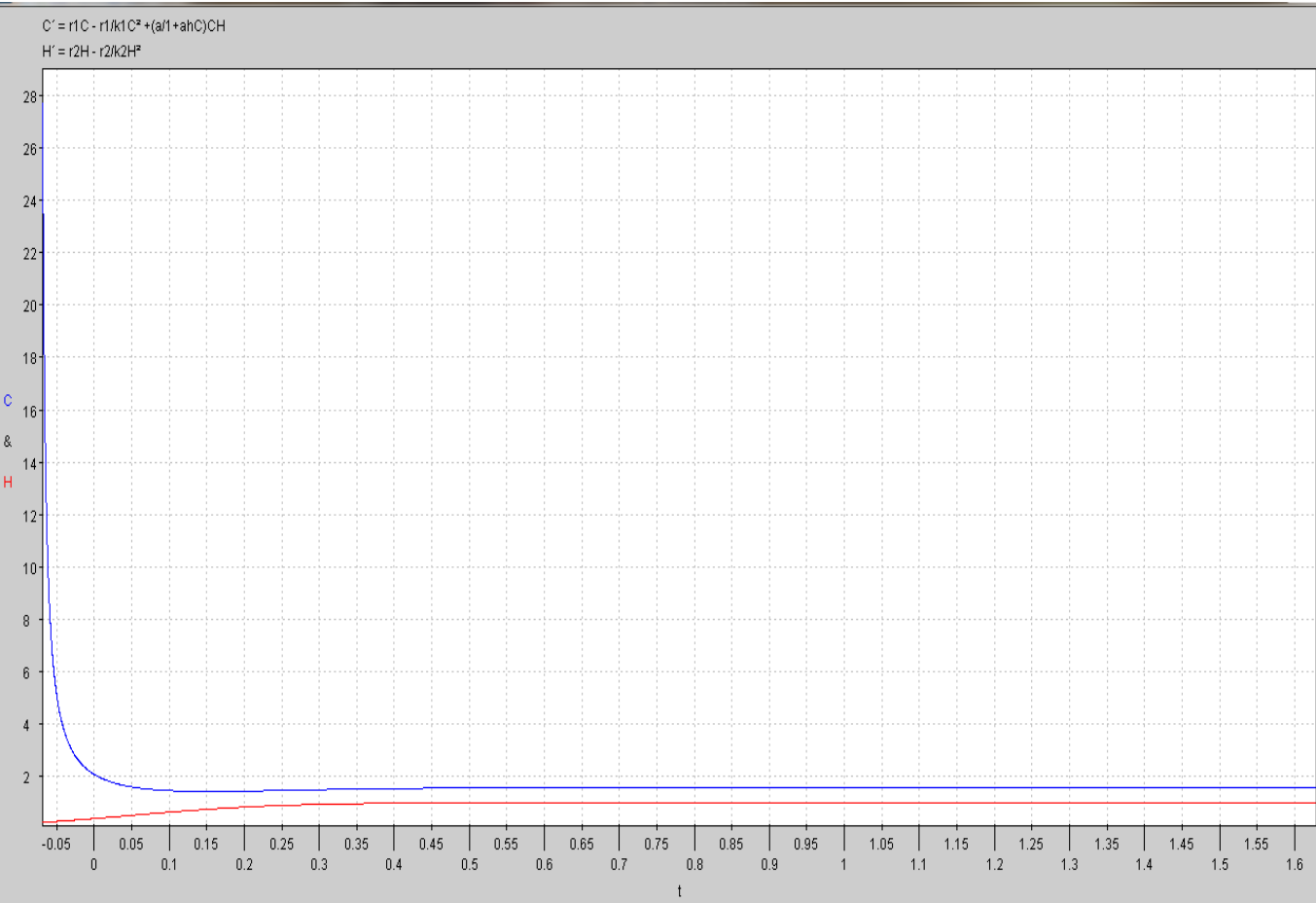

Figure 8. The Graph of the $\mathrm{C}-\mathrm{H}$ with Time $\mathrm{t}$ at the Equilibrium Point $\mathrm{S} 1$

The set of parameters are $\mathrm{r} 1=\mathbf{1 0}, \mathrm{r} 2=\mathbf{1 0}, \mathrm{k} 1=\mathbf{1}, \mathrm{k} 2=1, \mathrm{a}=\mathbf{5}, \mathrm{h}=\mathbf{0 . 0 9}$

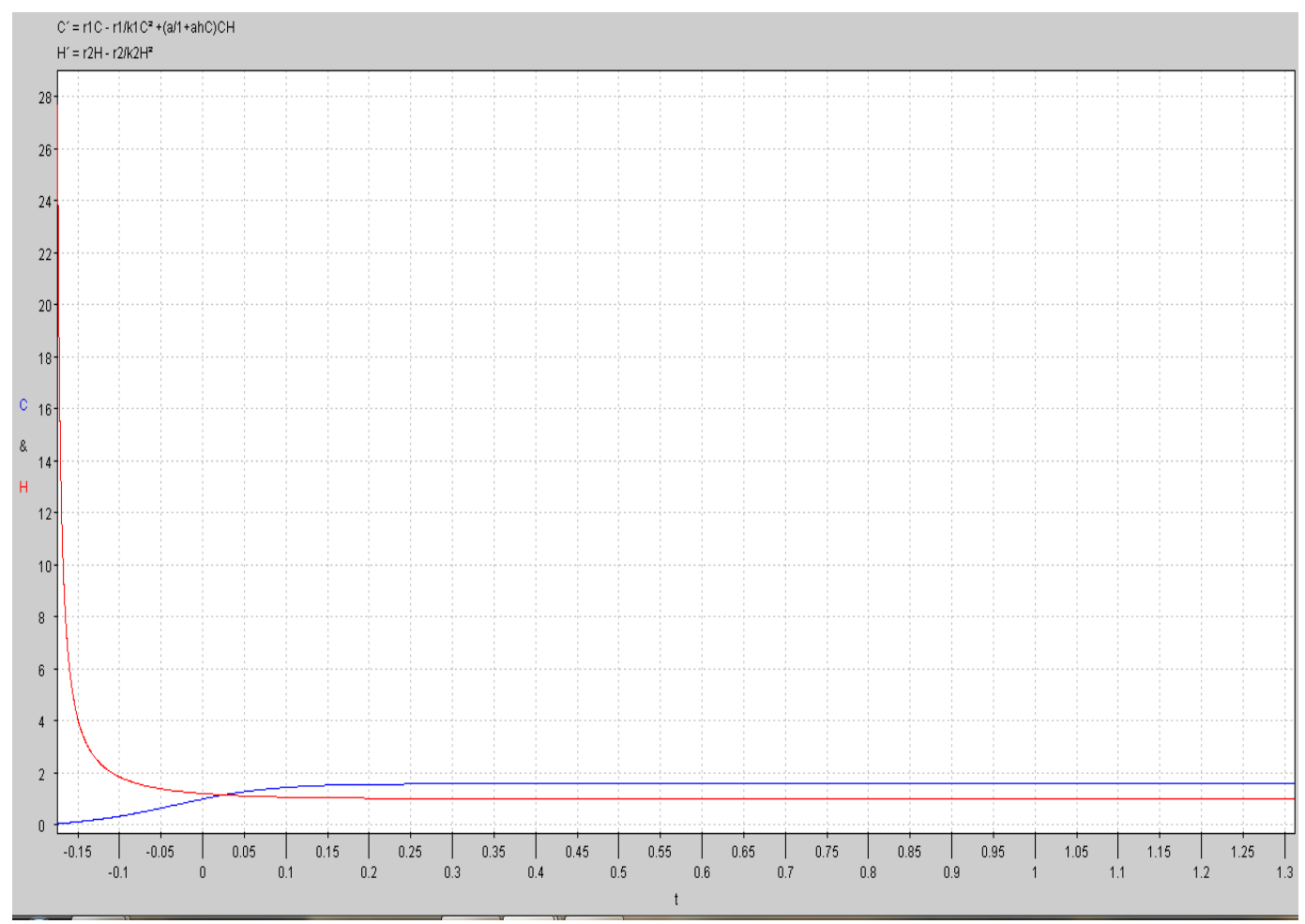

Figure 9. The Graph of the C- H with Time $\mathrm{t}$ at the Equilibrium Point S2

The set of parameters are $\mathrm{r} 1=\mathbf{1 0}, \mathrm{r} 2=\mathbf{1 0}, \mathrm{k} 1=\mathbf{1}, \mathrm{k} 2=\mathbf{1}, \mathbf{a}=\mathbf{5}, \mathbf{h}=\mathbf{0 . 0 9}$ 


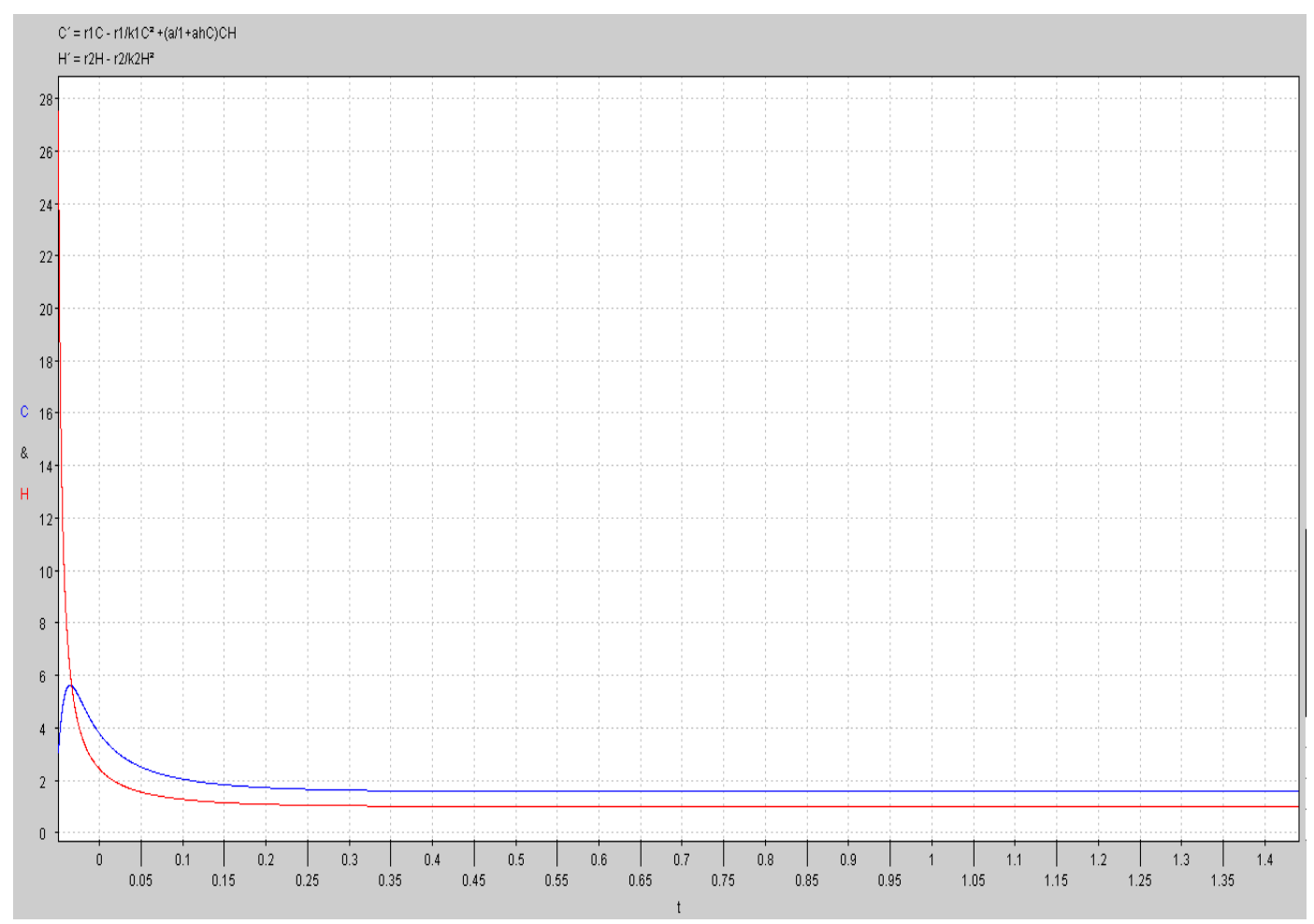

Figure 10. The Graph of the C- $\mathrm{H}$ with time $\mathrm{t}$ at the Equilibrium Point S3

The set of parameters are $r 1=\mathbf{1 0}, \mathbf{r} 2=10, k 1=1, k 2=1, a=5, h=0.09$

\section{Observations}

I) On increasing the value of $t$ for this set of parameters, it is observed that the equilibrium level of all the species of the system are increased or decreased. Furthermore, we can observe that the species will survive up to its maximum sustainability threshold without harming the others.

II) From Figure 1. we can observe that:

i. At $(0,0)$, both the species are fully washed out (vanishes)

ii. At $(k 1,0)$, only the commensal species exists

iii. At $(0, k 2)$, only the host species exists

iv. $\quad \operatorname{At}\left(\frac{k 1}{r 1}(r 1+a k 2), k 2\right)$,both species exist i.e co-existence state.

III) From Figure 6. one can observe that:

i. Equilibrium point $(0,0)$ is unstable in which the species does not exist.

ii. Equilibrium point $(1,0)$ is saddle in which the commensal species exists up to certain extent.

iii. Equilibrium point $(0,1)$ is saddle in which the host species exists without harmed.

iv. Equilibrium point $\left(\frac{\alpha-1+\sqrt{\alpha}}{2 \alpha}, 1\right)$ is stable (nodal sink)-co-existence.

\section{Results (Qualitative Analysis)}

(i). In first two models, when both the species were washed out, there is no significant interaction. 
(ii). In the second assumption sustainability of the commensal was determined without harming the host.

(iii). In the third assumption it is more relevant than the two assumptions.

\section{Conclusions}

(i). The commensalism relation is often between a larger host and a smaller commensal. The host organism is essentially unchanged by the interaction, whereas the commensal species may show great morphological adaptation.

(ii). This Model is a rudimentary model of the complex ecology of this world. It assumes just one Host for the commensal. It also assumes no outside influences like disease, changing conditions, pollution, and so on. However, the model can be expanded to include other variables.

(iii). We can polish the equations by adding more variables and get a better picture of the ecology. But with more variables, the model becomes more complex and would require more brains or computer resources.

(iv). This model is an excellent tool to explain the principals involved in ecology and also to show some adequate initiative results. It also shows a special relationship between biology and mathematics.

\section{References}

[1] W. Boyce, "Elementary Differential Equations", New York: John Wiley \& Sons, Inc., (1986).

[2] M. Michael and D. Zill, "Differential Equations with Boundary-Value Problems", Boston: PWS-Kent Publishing Company, (1993).

[3] Lotka Volterra Model. http://www.ento.vt.edu/sharov/PopEcol/lec10/lotka.html; 8/20/02

[4] Predator-Prey Modeling. http://www-rohan.sdsu.edu/ jmahaffy/courses/bridges/bridges00.

A. Hastings, "Global stability of two species Systems," Journal of Mathematical biology,

[5] N. Phanikumar, N. Seshagiri Rao and N. C. H. Pattabhi Ramacharyulu, "On the stability of a Host- A flourishing commensal species pair with limited resources", International Journal of Logic Based Intelligent Systems, vol. 3, no. 1, (2009), pp. 45-54. 